\title{
THE eRHIC RING-RING COLLIDER DESIGN
}

\author{
F. Wang, M. Farkhondeh, W. Franklin, W. Graves, R. Milner, J. Van der Laan, C. Tschalaer, \\ D. Wang, T. Zwart, A. Zolfaghari, MIT-Bates, Middleton, MA 01949, USA \\ J. Beebe-Wang, A. Deshpande, V. Litvinenko, W. W. Mackay, C. Montag, B. Parker, S. Peggs, \\ S. Ozaki, V. Ptitsyn, T. Roser, S. Tepikian, D. Trbojevic, BNL, Upton, NY, 11973, USA \\ D.P. Barber, DESY, 22607, Hamburg, Germany
}

\begin{abstract}
The eRHIC ring-ring collider is the main design option for the future lepton-ion collider at Brookhaven National Laboratory. The baseline design is presented in the eRHIC Zero ${ }^{\text {th }}$-Order Design Report (ZDR) [1]. We report on progress made during the past year including possible interaction region design changes, upgrade efforts on RHIC (the ion ring), and results of a new design study on the e-ring. The interaction region revision is based on a new scheme for the main detector design. RHIC upgrades are being pursued to ensure luminosity and ion polarization goals of the ring-ring option in the baseline design will be met. Higher current operation of the lepton storage ring is under serious consideration as a result of better understanding of beam-beam interaction effects.
\end{abstract}

\section{INTRODUCTION}

The basic design goals of the eRHIC ring-ring collider as outlined in the ZDR report are as follows:

- Provisions for electron collisions with protons, light nuclei and heavy nuclei,

- Collision energy range ( $\mathrm{E}_{\mathrm{cm}} /$ nucleon: 30-100 GeV),

- High luminosity ( $\mathrm{L}=4.4 \times 10^{32} \mathrm{~cm}^{-2} \mathrm{~s}^{-1}$ per nucleon, e-p),

- Polarization of electron and ion beams.

The ring-ring option is based on the relatively wellunderstood performance of storage rings and does not require extensive $\mathrm{R} \& \mathrm{D}$. Therefore the time scale of the collider project is relatively short and quite predictable. Many important nuclear physics experiments can be accomplished with the above design specifications.

A recent detector design study concluded that increasing the size of the region free of machine elements inside the detector would reduce background significantly [2]. A new interaction region design to accommodate this requirement has been worked out [3]. The resulting luminosity reduction and beam dynamic issues related to the new interaction region changes need to be addressed.

A study of beam-beam effects for the ring-ring options has raised the possibility of realizing higher proton beam beam-beam parameter. This would allow higher electron beam current in collision. Recent progress on an electron beam polarization study enables us to look at non-linear effects.

Finally, the continuing improvement on the RHIC operation and upgrade efforts will provide a solid foundation for a realistic site-specific design. The layout of the ZDR design of a 5-10 GeV electron (positron) ring intersects one ion ring (blue ring) in one point is shown in Figure 1.

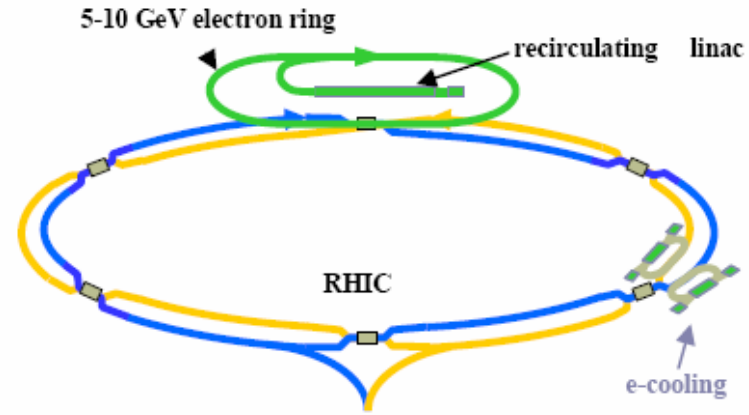

Figure 1: eRHIC ring-ring collider layout.

\section{INTERACTION REGION}

A new main detector design features a machine-element free region of $\pm 3 \mathrm{~m}$ around the IP. The new interaction region design must fulfill this requirement while dealing with other IP design challenges (merging and separation of beams, focusing, synchrotron radiation fan accommodation etc.). The optimal beam sizes at IP (for high luminosity) suggested by the new interaction regions design are twice as large as in the original design. Details of parameters changes and luminosity reduction in comparison to the ZDR design are listed in Table 1. Aside from luminosity reduction, the issue of greatest concern is the impact on the optics for the electron ring interaction straight section. The maximum electron beam $\beta$ function amplitudes near the IP will be several times larger, therefore resulting in much higher natural chromaticities for the e-ring. Electron ring chromaticity correction schemes and beam dynamic aperture issues must be readdressed.

Table 1: Summary of $\beta^{*}$ and luminosity changes.

\begin{tabular}{|l|l|l|l|l|}
\hline & \multicolumn{2}{|l|}{ e-p ZDR IP } & \multicolumn{2}{l|}{ e-p New IP } \\
\hline Beam & P & E & P & E \\
\hline $\begin{array}{l}\text { Bunch intensity } \\
10^{11}\end{array}$ & 1.0 & 1.0 & 1.0 & 1.0 \\
\hline $\begin{array}{l}\text { rms emittance, } \\
\mathrm{nm}, \mathrm{x} / \mathrm{y}\end{array}$ & $9.5 / 9.5$ & $53 / 9.5$ & $9.5 / 9.5$ & $53 / 9.5$ \\
\hline$\beta^{*}, \mathrm{~cm}, \mathrm{x} . / \mathrm{y}$ & $104 / 27$ & $19 / 27$ & $216 / 54$ & $38 / 54$ \\
\hline $\begin{array}{l}\text { Beam-beam } \\
\text { Parameters, } \mathrm{x} / \mathrm{y}\end{array}$ & 0.0065 & 0.027 & 0.0065 & 0.027 \\
\hline $\begin{array}{l}\text { Luminosity, } \\
1.0 \mathrm{e}^{32} \mathrm{~cm}^{-2} \mathrm{~s}^{-1}\end{array}$ & \multicolumn{3}{|c|}{4.4} & \multicolumn{2}{|c|}{2.003} \\
\hline
\end{tabular}




\section{ION RING UPGRADES}

The highest calculated luminosities for eRHIC assume several upgrades for existing RHIC ion rings. These include an increase in total ion beam intensity as well as improved beam emittance and polarization.

Since the single ion bunch intensity for the eRHIC ringring option is limited by electron beam-beam limit, the total intensity increase can be done only by increasing the number of circulating ion bunches. Present RHIC ion-ion and proton-proton operations use 56 bunches per ring. In upcoming years, a gradual increase of the number of bunches for RHIC operation to 112 is planned. At the same time, beam experimental and theoretical studies are underway to evaluate further increases of the number of bunches, with an ultimate goal of 360-bunch operation mode. The main areas of the evaluation here are electron cloud and pressure rise problems, which have been observed already at the existing intensities in most warm and a few cold sections of the rings. Possible remedies have been under intensive testing, like NEG vacuum chamber coating, in-situ baking, using solenoids, and antigrazing ridges on vacuum pipe [4]. This year, beam experiments demonstrated real advantages of pipe sections with NEG coating against the pressure rise. To go beyond 180 bunches the injection system upgrade will be required. It may include very fast injector kickers or injection schemes based on long flattop injection kickers or barrier rf stacking. Higher beam intensity will also require an upgraded abort system and a precise evaluation of heat load in the cryogenic beam pipe of the ion rings.

Polarized protons have been accelerated in RHIC to 100 $\mathrm{GeV}$, where the polarized proton experiment program is presently underway [5]. The RHIC rings utilize Siberian Snakes, two per ring, in order to preserve the beam polarization during the acceleration. Tests of polarized proton acceleration to higher energies, initially $205 \mathrm{GeV}$, then $250 \mathrm{GeV}$, are planned in upcoming years. Also upgrades in injector chain, most importantly the installation of a cold partial snake in AGS, should increase polarization level from $50 \%$ to $70 \%$. Helical spin rotators have been successfully used at RHIC operation to create longitudinal polarization in two of RHIC interaction points. The same rotator design will be used for the rotators around e-p collision region.

Besides the protons, polarized ${ }^{3} \mathrm{He}$ ions are being considered for experiments at eRHIC. The preservation of $\mathrm{He}$ ion polarization during acceleration is an important topic. Existing helical Siberian Snakes should work well for He beams too. However, the spin resonances for $\mathrm{He}$ ions are stronger and more numerous than for protons. Therefore detailed studies on polarized He acceleration are needed, including source development and polarization preservation in the injector chain.

Though ion cooling has been not part so far in the RHIC operation, electron cooling and stochastic cooling R\&D programs are underway. Electron cooling would bring and maintain ion transverse emittances considerably below typical values used during present operation. It will allow maximizing luminosity for e-Au collisions at the level allowed by beam-beam effects. The cooling of lower energy protons $(<150 \mathrm{GeV})$ would be also required to achieve high eRHIC e-p luminosities. For the proton case the cooling is considerably less efficient and would be done at the injection energy with consequent cooled beam acceleration. Continuing development and testing of different components of electron cooling system is underway [6]. Stochastic cooling is also being under development as a possible tool for maintaining of longitudinal beam emittance against intra-beam scattering.

\section{THE ELECTRON RING}

\section{Beam-Beam Effect}

Recent studies on beam-beam effects are detailed in [7] [8]. The first important result is about beam-beam limits, which set the current limits for electron or ion beam in collision. The simulation in reference [8] is done with $5 \times 10^{5}$ particles in each electron and proton bunch by using the particle-in-cell method. The beam-beam limits were examined as thresholds of the onset of coherent beam-beam instability. The proton beam-beam parameter $\xi_{\mathrm{p}}$ is 0.0065 in ZDR, based on RHIC operating experience and three IPs (one for e-p). In a dedicated mode with one IP, there is a possibility of colliding with higher proton beam-beam parameters, which in turn requires higher electron beam current and results in higher luminosity. This is shown in Figure 2. The $\xi_{\mathrm{p}}$ value close to the threshold of the coherent beam-beam instability is about 0.015 which corresponding to 2.3 times higher electron bunch intensity than ZDR design. Electron beam beambeam parameter of 0.08 in the baseline design is close to the limit predicted by these simulations.

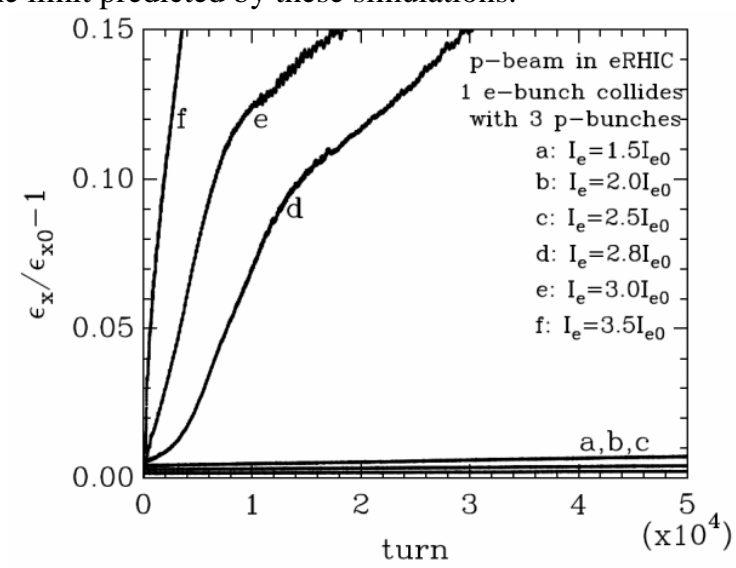

Figure 2: Proton beam emittance growth vs. electron bunch intensity, where $\varepsilon_{x 0}$ and $\mathrm{I}_{\mathrm{e} 0}$ are baseline design values, $\mathrm{I}_{\mathrm{e}}$ is the bunch current used in the simulations.

Another study has examined coherent beam-beam effects in a collider consisting of two rings of unequal circumference. There are concerns that such a design could diminish the stable operation area in the tune diagram [9]. No coherent instability occurs for eRHIC 
with the 1:3 circumference ratio in these simulations. In fact, the large tune spreads associated with the high beambeam parameters are the main concerns of partial luminosity reductions. Choosing appropriate working points, which should be different for $\mathrm{e}^{+}$and $\mathrm{e}^{-}$as the tune spread direction differs, will largely solve the problem.

\section{Electron Beam Polarization}

The early calculations [1] [10] of the attainable $\mathrm{e}^{+-}$ equilibrium polarization were made with the SLIM algorithm [11] with the code SLICK. It was shown that with good magnet alignment and orbit correction, equilibrium polarizations of up to about 80 percent should be possible at $10 \mathrm{GeV}$ in the absence of beam-beam interactions (The electron beam would be prepolarized before injection). However, the SLIM formalism linearizes the spin and orbital motion when calculating the effects of the stochastic photon emission, which leads to depolarization. Therefore, it can only predict the strength of first-order depolarizing resonances.

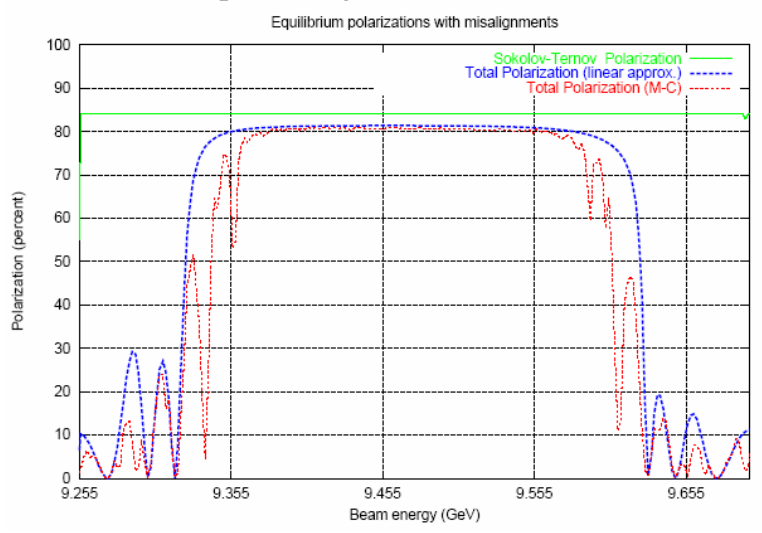

Figure 3: Equilibrium polarization (linear approx. and MC simulations).

The best way go beyond the linearization and to include the effects of nonlinear orbit motion and beam-beam forces, is to simulate the effects of stochastic photon emission in a Monte-Carlo (M-C) spin-orbit tracking code which operates on a large ensemble of particles and includes the full 3-D spin motion. See also [12]. The M-C algorithm has being implemented in the code SLICKTRACK, an extended version of SLICK. A sample result for the equilibrium polarization, in the range 9.255$9.697 \mathrm{GeV}$ is shown in Figure 3, together with the curve for the corresponding linear approximation. One can now see the expected higher order resonances. They are essentially the so-called synchrotron sidebands of the first order resonances and it is clear that with the inclusion of full 3-D spin motion, high polarizations are still attainable. The next step, which is well underway, is the inclusion of beam-beam forces.

\section{Modular Dipole for Low Energy Operation}

To ensure a wide range of collision energies, the electron ring must be operable from $5 \mathrm{GeV}$ to $10 \mathrm{GeV}$. In an electron storage ring, synchrotron radiation intensity, which is proportional to the $4^{\text {th }}$ power of energy, defines beam emittance. It also dominates a number of dynamic processes including beam polarization, damping of instabilities, and balance of beam-beam effect. Enhanced synchrotron radiation is the key to retain high beam-beam limits, and therefore, high luminosity in low energy operations [1]. A modular dipole concept which could support convenient adjustment of radiation strength is preferable to a more complicated radiation wiggler scheme [13]. Figure 4 shows beam central orbit shifts in a three pieces modular dipole magnet. The beam-beam limit reduction from $10 \mathrm{GeV}$ to $5 \mathrm{GeV}$ will be $20 \%$ instead of $50 \%$ in the baseline design. In the eRHIC injector, positron beam is unpolarized, so the self-polarization mechanism is employed for positron beam polarization. With this scheme, the self-polarization time of e+ at $5 \mathrm{GeV}$ is one hour, extending the availability of polarized positron beam to experiments at $5 \mathrm{GeV}$.

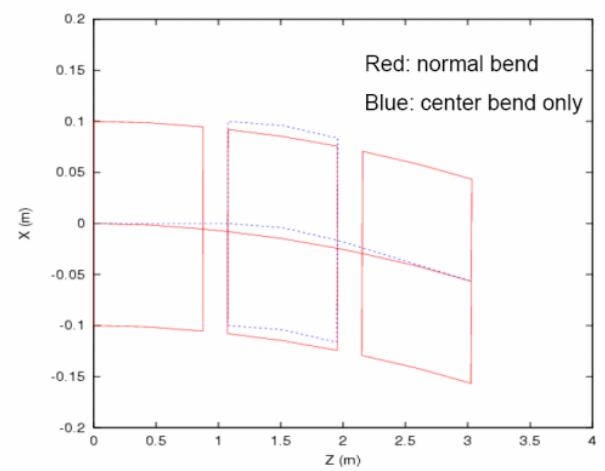

Figure 4: Beam orbit shift in a modular dipole magnet.

\section{REFERENCES}

[1] M. Farkhondeh and V.Ptitsyn edit., BNL C-AD note 1422004.

[2] B. Surrow, "eRHIC Detector Design Studies", these proceedings.

[3] C. Montag, et al., "Interaction region design for the electron-ion collider eRHIC", these proceedings.

[4] S.Y. Zhang et al., "Beam Induced Pressure Rise at RHIC", these proceedings.

[5] M. Bai, et al., "Polarized Proton Collisions at RHIC", these proceedings.

[6] I. Ben-Zvi et al., "Electron Cooling of RHIC", these proceedings.

[7] C. Montag, "Beam-beam simulations for the eRHIC Electron Ring", these proceedings.

[8] J. Shi et al., "Study of Beam-Beam effects in eRHIC with self-consistent B-B simulations", these proceedings.

[9] K. Hirata and E. Keil, CERN/LEP-TH/89-54.

[10] F. Wang, et al., EPAC'04, Lucerne, July 2004, p.887.

[11] A. Chao \& M. Tigner edit. "Handbook of Accel. Phys. and Eng.", $2^{\text {nd }}$ Edit., p. 159.

[12] J. Kewisch, T. Limberg, R. Rossmanith, Phys. Rev. Lett., vol.62, p.419, (1989).

[13] F. Wang, "Luminosity Considerations for eRHIC", second Electron-Ion Collider Workshop, JLab, March $15-17,2004$ 